\title{
On 2D integro-differential systems. Stability and sensitivity analysis
}

\author{
Monika Bartkiewicz ${ }^{1}$ • Marek Majewski ${ }^{1}$ (D) \\ Stanisław Walczak ${ }^{1,2}$
}

\begin{abstract}
In the paper a two-dimensional integro-differential system is considered. Using some variational methods we give sufficient conditions for the existence and uniqueness of a solution to the considered system. Moreover, we show that the system is stable and robust.
\end{abstract}

Keywords 2D integro-differential system $\cdot$ Sensitivity $\cdot$ Robustness $\cdot$ Stability

\section{Introduction}

We will denote by $Q$ the unit interval in $\mathbb{R}^{2}$, i.e.

$$
Q=\left\{(x, y) \in \mathbb{R}^{2}: x \in[0,1] \text { and } y \in[0,1]\right\}
$$

General continuous 2D differential system has the following form

$$
\begin{aligned}
z_{x y}(x, y) & =f\left(x, y, z(x, y), z_{x}(x, y), z_{y}(x, y)\right), \\
z(x, 0) & =a(x), z(0, y)=b(y), a(0)=b(0),
\end{aligned}
$$

Marek Majewski

marmaj@math.uni.lodz.pl

Monika Bartkiewicz

bartkiew@math.uni.lodz.pl

Stanisław Walczak

stawal@math.uni.lodz.pl

1 Faculty of Mathematics and Computer Science, University of Lodz, Banacha 22, 90-238 Lodz, Poland

2 State School of Higher Vocational Education, Batorego 64 C, 96-100 Skierniewice, Poland 
where $f: Q \times \mathbb{R}^{n} \times \mathbb{R}^{n} \times \mathbb{R}^{n} \rightarrow \mathbb{R}^{n}, n \geq 1, a, b:[0,1] \rightarrow \mathbb{R}^{n}$ are given functions. The linear case of system (2) can be written as

$$
\begin{aligned}
z_{x y}(x, y)= & A_{0}(x, y) z(x, y)+A_{1}(x, y) z_{x}(x, y)+A_{2}(x, y) z_{y}(x, y) \\
& +w(x, y),
\end{aligned}
$$

where $A_{0}, A_{1}, A_{2}: \mathbb{R}^{2} \rightarrow \mathbb{R}^{n \times n}$ are given functions (which values are matrices of the dimension $n \times n), w$ is a given $n$-dimensional vector function and $z$ satisfies boundary conditions (3). Continuous 2D systems correspond to the discrete model of Fornasini-Marchesini type, which has the following form (see Fornasini and Marchesini 1976)

$$
\begin{gathered}
z(i+1, j+1)=A_{0}(i, j) z(i, j)+A_{1}(i, j) z(i+1, j)+A_{2}(i, j) z(i, j+1)+w(i, j), \\
z(i, 0)=a(i), z(0, j)=b(j), a(0)=b(0),
\end{gathered}
$$

$i, j=0,1,2, \ldots$

Two-dimensional discrete systems (5)-(6) and continuous systems (2)-(3) play an essential role in mathematical modeling of many technical, physical, biological and other phenomena. For example, in the paper by Fornasini (see Fornasini 1991) 2D space models of the form (5)-(6) were applied to the investigation of the process of pollution and self purification of a river. Application of the $2 \mathrm{D}$ discrete models to image processing and transmission were studied in the book of Bracewell (see Bracewell 1995). The 2D continuous systems of the form (2)-(3) were adopted to investigation of the gas filtration model (see Bors and Walczak 2012). Other applications of discrete and continuous 2D systems in the theory of automatic control, stability, robotics and optimization can be found in papers of Galkowski et al. (2003), Paszke et al. (2004), Lomadze et al. (2008), Kaczorek (2001), Dey and Kar (2011), Singh (2008), Idczak and Walczak (2000) and in the monograph of Kaczorek (1985).

In the paper, we investigate $2 \mathrm{D}$ integro-differential system of the form

$$
\begin{aligned}
& z_{x y}(x, y)+f^{1}(x, y, z(x, y))+\int_{0}^{x} \int_{0}^{y}\left(f^{2}(s, t, z(s, t))+A^{1}(s, t) z_{x}(s, t)\right. \\
& \left.+A^{2}(s, t) z_{y}(s, t)\right) d s d t=v(x, y)
\end{aligned}
$$

with the following boundary conditions

$$
z(x, 0)=0 \text { for } x \in[0,1] \text { and } z(0, y)=0 \text { for } y \in[0,1],
$$

where $f^{1}, f^{2}: Q \times \mathbb{R}^{n} \rightarrow \mathbb{R}, A^{1}, A^{2}: Q \rightarrow \mathbb{R}^{n} \times \mathbb{R}^{n}$ are given functions (for more details see Sect. 3). We shall consider the above system in the space of absolutely continuous functions of two variables. The definition and basic properties of absolutely continuous functions defined on the interval $Q$ are presented in Sect. 2.

In the paper we prove, under assumptions (C1)-(C3) (see Sect. 3), that for any square integrable function $v$ system (7)-(8) possesses a unique solution $z_{v}$ which continuously depends on $v$ and the operator $v \mapsto z_{v}$ is differentiable in the Fréchet sense, i.e. the considered system is well-posed and robust. It should be noticed that robustness can be understood in many aspects and meanings. However, usually it is the ability of a system to resist change without adapting its initial stable configuration Fukuda (2015). If the operator $u \mapsto z_{v}$ is differentiable then the change of the solution caused by the change of control $v$ is not rapid. The speed of this change can be expressed by the derivative of the operator $u \mapsto z_{v}$, which measures the resistance of the system to the change of $v$. 
The proof of the main result, given in Sect. 4, is based on the global diffeomorphism theorem (Theorem 1). In the final part of the paper we give an example and compare the method used in the paper with the methods based on the contraction principle and the Schauder fixed point theorem.

\section{Preliminaries}

In this section we introduce some mathematical backgrounds necessary to understand further considerations. In particular, we define the space of absolutely continuous functions which is the space of solutions and present its basic properties.

We begin with the following theorem on a diffeomorphism between Banach and Hilbert spaces which is the main tool in the proof of the main result.

Theorem 1 (see Idczak and Skowron 2012) Let $Z$ be a real Banach space, $V$ be a real Hilbert space, $F: Z \rightarrow V$ be an operator of $C^{1}$ class. If

(a) for any $v \in V$ the functional $\varphi(z)=\frac{1}{2}\|F(z)-v\|_{V}^{2}$ satisfies Palais-Smale condition ((PS)-condition),

(b) for any $v \in V$ the equation $F^{\prime}(z) h=v$ possesses a unique solution,

then

1. for any $v \in V$ there exists exactly one solution $z_{v} \in Z$ to the system $F(z)=v$,

2. the operator $V \ni v \rightarrow z_{v} \in Z$ is differentiable in the Fréchet sense.

In other words, the operator $F$ is a diffeomorphism between Banach space $Z$ and Hilbert space $V$.

We recall that functional $\phi$ satisfies (PS)-condition if whenever there is a sequence $\left\{z^{n}\right\} \subset Z$ with $\left|\phi\left(z^{n}\right)\right| \leq$ const and $\phi^{\prime}\left(z^{n}\right) \rightarrow 0$ in the space $Z^{*}$ of linear and continuous functionals defined on $Z$, then in the closure of the set $\left\{z^{n}: n \in N\right\}$, there is some point $\bar{z}$ where $\phi^{\prime}(\bar{z})=0$ (see Aubin and Ekeland 2006).

From the bounded inverse theorem (see Limaye 1996, Theorem 11.1) it follows that for any $z \in Z$ there exists a constant $\alpha_{z}>0$ such that $\left\|F^{\prime}(z) h\right\|_{V} \geq \alpha_{z}\|h\|_{z}$. Therefore it follows easily that the above theorem is equivalent to Idczak and Skowron (2012, Theorem 3.1), with $f=F$.

Let us denote by $A C\left(Q, \mathbb{R}^{n}\right)$ the space of absolutely continuous vector functions $z=$ $\left(z_{1}, z_{2}, \ldots, z_{n}\right)$ defined on the interval $Q$. The geometrical definition of the space $A C(Q, \mathbb{R})$ can be found in papers Berkson and Gillespie (1984) and Walczak (1987). In this paper we need necessary and sufficient conditions for $z: Q \rightarrow \mathbb{R}^{n}$ to be absolutely continuous on $Q$ i.e. $z \in A C\left(Q, \mathbb{R}^{n}\right)$. We have the following theorem (see Berkson and Gillespie 1984; Walczak 1987).

Theorem 2 A function z belongs to the space $A C\left(Q, \mathbb{R}^{n}\right)$ if and only if there exist functions $l \in L^{1}\left(Q, \mathbb{R}^{n}\right), l^{1}, l^{2} \in L^{1}\left([0,1], \mathbb{R}^{n}\right)^{1}$ and a constant $c \in \mathbb{R}^{n}$ such that

$$
z(x, y)=\int_{0}^{x} \int_{0}^{y} l(s, t) d s d t+\int_{0}^{x} l^{1}(s) d s+\int_{0}^{y} l^{2}(t) d t+c .
$$

${ }^{1}$ Throughout the paper $L^{p}\left(Q, \mathbb{R}^{n}\right), p \geq 1$ denotes the space of all functions $z: Q \rightarrow \mathbb{R}^{n}$ which are measurable and $\int_{Q}|z(z)|^{p} d x<\infty$ 
Moreover the function $z$ possesses partial derivatives $z_{x}, z_{y}, z_{x y}$, for a.e. $(x, y) \in Q$ and

$$
\begin{aligned}
z_{x}(x, y) & =\int_{0}^{y} l(x, t) d t+l^{1}(x), \\
z_{y}(x, y) & =\int_{0}^{x} l(s, y) d s+l^{2}(y), \\
z_{x y}(x, y) & =l(x, y) .
\end{aligned}
$$

Theorem 2 follows directly from (Berkson and Gillespie 1984, Theorem 4) and (Šremr 2010, Proposition 3.5) (see also (Walczak 1987, Theorem 2), (Walczak 1998, Theorem 1)).

It is easy to check that if the function $z$ satisfies homogeneous boundary conditions, i.e. $z(x, 0)=0$ for $x \in[0,1]$ and $z(0, y)=0$ for $y \in[0,1]$ then $l^{1}=0, l^{2}=0, c=0$ and consequently we can write

$$
z(x, y)=\int_{0}^{x} \int_{0}^{y} l(s, t) d s d t=\int_{0}^{x} \int_{0}^{y} z_{x y}(s, t) d s d t .
$$

By $A C_{0}^{2}\left(Q, \mathbb{R}^{n}\right)$ we shall denote the space of absolutely continuous functions on the interval $Q$ which satisfy the homogeneous boundary conditions $z(x, 0)=z(0, y)=0$ for $x, y \in[0,1]$ and such that $z_{x y} \in L^{2}\left(Q, \mathbb{R}^{n}\right)$. The space $A C_{0}^{2}$ is a Hilbert space with the inner product given by formula

$$
\langle z, \bar{z}\rangle=\int_{0}^{1} \int_{0}^{1}\left\langle z_{x y}(x, y), \bar{z}_{x y}(x, y)\right\rangle d x d y .
$$

In the space $A C_{0}^{2}\left(Q, \mathbb{R}^{n}\right)$ we introduce two norms. The first one is a classical norm given by the formula

$$
\|z\|=\left(\int_{0}^{1} \int_{0}^{1}\left|z_{x y}(x, y)\right|^{2} d x d y\right)^{\frac{1}{2}}=\left\|z_{x y}\right\|_{L^{2}}
$$

and the second one is defined by the integral with exponential weight

$$
\|z\|_{A C_{0, m}^{2}}=\left(\int_{0}^{1} \int_{0}^{1} e^{-m(x+y)}\left|z_{x y}(x, y)\right|^{2} d x d y\right)^{\frac{1}{2}}, m>0 .
$$

Exponential norm (12) was introduced by Bielecki (1956). The space $A C_{0}^{2}\left(Q, \mathbb{R}^{n}\right)$ with norm (12) will be denoted by $A C_{0, m}^{2}\left(Q, \mathbb{R}^{n}\right)$.

It is easy to notice that

$$
e^{-2 m}\|z\| \leq\|z\|_{A C_{0, m}^{2}} \leq\|z\| .
$$

Thus the norms given by formulas (11) and (12) are equivalent.

Similarly, in the space $L^{2}\left(Q, \mathbb{R}^{n}\right)$ of square integrable functions on $Q$ we introduce two equivalent norms:

$$
\|v\|_{L^{2}}=\left(\int_{0}^{1} \int_{0}^{1}|v(x, y)|^{2} d x d y\right)^{\frac{1}{2}}
$$

and

$$
\|v\|_{L_{m}^{2}}=\left(\int_{0}^{1} \int_{0}^{1} e^{-m(x+y)}|v(x, y)|^{2} d x d y\right)^{\frac{1}{2}} .
$$

The space of square integrable functions with norm (13) will be denoted by $L_{m}^{2}\left(Q, \mathbb{R}^{n}\right)$. 


\section{Basic assumptions and lemmas}

In this section we formulate basic assumptions and proves lemmas. Lemma 1 has a technical character, whereas Lemmas 2 and 3 are fundamental for understanding the proof of the main theorem-Theorem 3.

On the functions defining system (7) we assume that

(C1) the functions $f^{1}(\cdot, \cdot, z), f^{2}(\cdot, \cdot, z)$ are measurable on $Q$ for every $z \in \mathbb{R}^{n}$ and $f^{1}(x, y, \cdot), f^{2}(x, y, \cdot)$ are continuously differentiable on $\mathbb{R}^{n}$ for a.e. $(x, y) \in Q$, the function $A^{1}(\cdot, y)$ is differentiable for a.e. $y \in[0,1]$, the function $A^{2}(x, \cdot)$ is differentiable for a.e. $x \in[0,1]$, the functions $A^{1}, A^{2}, A_{x}^{1}, A_{y}^{2}$ are measurable on $Q$ and essentially bounded on $Q$, the function $v \in L^{2}\left(Q, \mathbb{R}^{n}\right)$;

(C2) there exist a constant $B>0$ and a function $b \in L^{2}\left(Q, \mathbb{R}^{+}\right)$such that

$$
\left|f^{1}(x, y, z)\right|,\left|f^{2}(x, y, z)\right| \leq B|z|+b(x, y)
$$

and

$$
\left|A^{1}(x, y)\right|,\left|A^{2}(x, y)\right|,\left|A_{x}^{1}(x, y)\right|,\left|A_{y}^{2}(x, y)\right| \leq B
$$

for $z \in \mathbb{R}^{n}$ and a.e. $(x, y) \in Q$;

(C3) the functions $f_{z}^{1}, f_{z}^{2}$ are bounded on bounded sets, i.e. for any $\varrho>0$ there exists a constant $M_{\varrho}$, such that

$$
\left|f_{z}^{1}(x, y, z)\right|,\left|f_{z}^{2}(x, y, z)\right| \leq M_{\varrho}
$$

for $(x, y) \in Q$ and $|z| \leq \varrho$.

In the following lemma we prove some estimates for functions from the space $A C_{0}^{2}\left(Q, \mathbb{R}^{n}\right)$.

Lemma 1 If the function $z \in A C_{0}^{2}\left(Q, \mathbb{R}^{n}\right)$ then

$$
\begin{aligned}
\|z\|_{L_{m}^{2}} & \leq \frac{2}{m}\|z\|_{A C_{0, m}^{2}}, \\
\left\|w_{0}\right\|_{L_{m}^{2}} & \leq \frac{2}{m}\|z\|_{A C_{0, m}^{2}}, \\
\left\|w_{1}\right\|_{L_{m}^{2}} & \leq \frac{2}{m}\|z\|_{A C_{0, m}^{2}}, \\
\left\|w_{2}\right\|_{L_{m}^{2}} & \leq \frac{2}{m}\|z\|_{A C_{0, m}^{2}},
\end{aligned}
$$

where $w_{0}(x, y)=\int_{0}^{x} \int_{0}^{y}|z(s, t)| d s d t, \quad w_{1}(x, y)=\int_{0}^{x} \int_{0}^{y}\left|z_{x}(s, t)\right| d s d t$, $w_{2}(x, y)=\int_{0}^{x} \int_{0}^{y}\left|z_{y}(s, t)\right| d s d t$.

Remark 1 The norms $\|\cdot\|_{L_{m}^{2}}$ and $\|\cdot\|_{A C_{0, m}^{2}}$ are defined by (12) and (13) respectively. 
Proof Let $z$ be an arbitrary function from the space $A C_{0}^{2}\left(Q, \mathbb{R}^{n}\right)$. By (9) and applying Cauchy-Schwarz inequality we get

$$
\begin{aligned}
\|z\|_{L_{m}^{2}}^{2} & =\int_{0}^{1} \int_{0}^{1} e^{-m(x+y)}|z(x, y)|^{2} d x d y \\
& \leq \int_{0}^{1} \int_{0}^{1} e^{-m(x+y)}\left(\int_{0}^{x} \int_{0}^{y}\left|z_{x y}(s, t)\right| d s d t\right)^{2} d x d y \\
& \leq \int_{0}^{1} \int_{0}^{1}\left(e^{-m(x+y)} \int_{0}^{x} \int_{0}^{y}\left|z_{x y}(s, t)\right|^{2} d s d t \cdot \int_{0}^{x} \int_{0}^{y} d s d t\right) d x d y \\
& \leq \int_{0}^{1} \int_{0}^{1}\left(e^{-m(x+y)} \int_{0}^{x} \int_{0}^{y}\left|z_{x y}(s, t)\right|^{2} d s d t\right) d x d y .
\end{aligned}
$$

Applying integrating by parts formula for the inner integral (with respect to $x$ ) we obtain

$$
\begin{aligned}
\int_{0}^{1} & \int_{0}^{1}\left(e^{-m(x+y)} \int_{0}^{x} \int_{0}^{y}\left|z_{x y}(s, t)\right|^{2} d s d t\right) d x d y \\
& =\int_{0}^{1}\left(\int_{0}^{1}\left(e^{-m(x+y)} \int_{0}^{x} \int_{0}^{y}\left|z_{x y}(s, t)\right|^{2} d s d t\right) d x\right) d y \\
& =\int_{0}^{1}\left(\left[\frac{-1}{m} e^{-m(x+y)} \int_{0}^{x} \int_{0}^{y}\left|z_{x y}(s, t)\right|^{2} d s d t\right]_{x=0}^{x=1}\right. \\
& \left.-\int_{0}^{1}\left(\frac{-1}{m} e^{-m(x+y)} \int_{0}^{y}\left|z_{x y}(x, t)\right|^{2} d t\right) d x\right) d y \\
& =\int_{0}^{1}\left(\frac{-1}{m} e^{-m(1+y)} \int_{0}^{1} \int_{0}^{y}\left|z_{x y}(s, t)\right|^{2} d s d t\right) d y \\
& +\int_{0}^{1}\left(\int_{0}^{1}\left(\frac{1}{m} e^{-m(x+y)} \int_{0}^{y}\left|z_{x y}(x, t)\right|^{2} d t\right) d x\right) d y .
\end{aligned}
$$

Changing the order of integrals (thanks to Fubini's theorem) and again applying integrating by parts formula (for the integrals with respect to $y$ ), we get that the last term in (18) equals to

$$
\begin{aligned}
& \int_{0}^{1}\left(\frac{-1}{m} e^{-m(1+y)} \int_{0}^{y}\left(\int_{0}^{1}\left|z_{x y}(s, t)\right|^{2} d s\right) d t\right) d y \\
& +\int_{0}^{1}\left(\int_{0}^{1}\left(\frac{1}{m} e^{-m(x+y)} \int_{0}^{y}\left|z_{x y}(x, t)\right|^{2} d t\right) d y\right) d x \\
& =\left[\frac{1}{m^{2}} e^{-m(1+y)} \int_{0}^{y} \int_{0}^{1}\left|z_{x y}(s, t)\right|^{2} d s d t\right]_{y=0}^{y=1} \\
& +\int_{0}^{1}\left(\frac{1}{m^{2}} e^{-m(1+y)} \int_{0}^{1}\left|z_{x y}(s, y)\right|^{2} d s\right) d y \\
& +\int_{0}^{1}\left(\left[\frac{1}{m^{2}} e^{-m(x+y)} \int_{0}^{y}\left|z_{x y}(x, t)\right|^{2} d t\right]_{y=0}^{y=1}\right. \\
& \left.+\int_{0}^{1} \frac{1}{m^{2}} e^{-m(x+y)}\left|z_{x y}(x, y)\right|^{2} d y\right) d x
\end{aligned}
$$




$$
\begin{aligned}
& =\frac{1}{m^{2}} e^{-2 m} \int_{0}^{1} \int_{0}^{1}\left|z_{x y}(s, t)\right|^{2} d s d t+\frac{1}{m^{2}} \int_{0}^{1} \int_{0}^{1} e^{-m(1+y)}\left|z_{x y}(s, y)\right|^{2} d s d y \\
& +\frac{1}{m^{2}} \int_{0}^{1} \int_{0}^{1} e^{-m(x+1)}\left|z_{x y}(x, t)\right|^{2} d t d x+\frac{1}{m^{2}} \int_{0}^{1} e^{-m(x+y)}\left|z_{x y}(x, y)\right|^{2} d y d x \\
& \leq \frac{4}{m^{2}} \int_{0}^{1} \int_{0}^{1} e^{-m(x+y)}\left|z_{x y}(x, t)\right|^{2} d x d y=\frac{4}{m^{2}}\|z\|_{A C_{0, m}^{2}}^{2} .
\end{aligned}
$$

We have thus proved inequality (14). Making use of (9) and from what has already been proved, it follows that

$$
\begin{aligned}
\left\|w_{0}\right\|_{L_{m}^{2}}^{2} & =\int_{0}^{1} \int_{0}^{1} e^{-m(x+y)}\left(\int_{0}^{x} \int_{0}^{y}|z(s, t)| d s d t\right)^{2} d x d y \\
& \leq \int_{0}^{1} \int_{0}^{1} e^{-m(x+y)}\left(\int_{0}^{x} \int_{0}^{y}\left(\int_{0}^{s} \int_{0}^{t}\left|z_{x y}(\sigma, \tau)\right| d \sigma d \tau\right) d s d t\right)^{2} d x d y \\
& \leq \int_{0}^{1} \int_{0}^{1} e^{-m(x+y)}\left(\int_{0}^{x} \int_{0}^{y}\left(\int_{0}^{x} \int_{0}^{y}\left|z_{x y}(\sigma, \tau)\right| d \sigma d \tau\right) d s d t\right)^{2} d x d y \\
& \leq \int_{0}^{1} \int_{0}^{1} e^{-m(x+y)}\left(\int_{0}^{x} \int_{0}^{y}\left|z_{x y}(\sigma, \tau)\right| d \sigma d \tau \cdot \int_{0}^{x} \int_{0}^{y} d s d t\right)^{2} d x d y \\
& \leq \int_{0}^{1} \int_{0}^{1} e^{-m(x+y)}\left(\int_{0}^{x} \int_{0}^{y}\left|z_{x y}(\sigma, \tau)\right| d \sigma d \tau\right)^{2} d x d y \leq \frac{4}{m^{2}}\|z\|_{A C_{0, m}^{2}}^{2} .
\end{aligned}
$$

Let us prove the next inequality. By (9) and by already proved inequality we have

$$
\begin{aligned}
\left\|w_{1}\right\|_{L_{m}^{2}}^{2} & =\int_{0}^{1} \int_{0}^{1} e^{-m(x+y)}\left(\int_{0}^{x} \int_{0}^{y}\left|z_{x}(s, t)\right| d s d t\right)^{2} d x d y \\
& \leq \int_{0}^{1} \int_{0}^{1} e^{-m(x+y)}\left(\int_{0}^{x} \int_{0}^{y}\left(\int_{0}^{t}\left|z_{x y}(s, \tau)\right| d \tau\right) d s d t\right)^{2} d x d y \\
& \leq \int_{0}^{1} \int_{0}^{1} e^{-m(x+y)}\left(\int_{0}^{y}\left(\int_{0}^{x} \int_{0}^{t}\left|z_{x y}(s, \tau)\right| d \tau d s\right) d t\right)^{2} d x d y \\
& \leq \int_{0}^{1} \int_{0}^{1} e^{-m(x+y)}\left(\int_{0}^{y}\left(\int_{0}^{x} \int_{0}^{y}\left|z_{x y}(s, \tau)\right| d \tau d s\right) d t\right)^{2} d x d y \\
& \leq \int_{0}^{1} \int_{0}^{1} e^{-m(x+y)}\left(\int_{0}^{x} \int_{0}^{y}\left|z_{x y}(s, \tau)\right| d \tau d s \cdot \int_{0}^{y} d t\right)^{2} d x d y \\
& \leq \int_{0}^{1} \int_{0}^{1} e^{-m(x+y)}\left(\int_{0}^{x} \int_{0}^{y}\left|z_{x y}(s, t)\right| d s d t\right)^{2} d x d y=\left\|w_{0}\right\|_{L_{m}^{2}}^{2} \leq \frac{4}{m^{2}}\|z\|_{A C_{0, m}^{2}}^{2} .
\end{aligned}
$$

We conclude similarly that

$$
\left\|w_{2}\right\|_{L_{m}^{2}}^{2} \leq \frac{4}{m^{2}}\|z\|_{A C_{0, m}^{2}}
$$

Denote by $F: A C_{0}^{2}\left(Q, \mathbb{R}^{n}\right) \rightarrow L^{2}\left(Q, \mathbb{R}^{n}\right)$ the operator:

$$
\begin{aligned}
F(z)(x, y)= & z_{x y}(x, y)+f^{1}(x, y, z(x, y)) \\
& +\int_{0}^{x} \int_{0}^{y}\left(f^{2}(s, t, z(s, t))+A^{1}(s, t) z_{x}(s, t)+A^{2}(s, t) z_{y}(s, t)\right) d s d t .
\end{aligned}
$$


We will prove that the norm of $F$ is coercive.

Lemma 2 If the functions $f^{1}, f^{2}, A^{1}, A^{2}$ satisfy assumptions $(\boldsymbol{C 1})$ and $(\boldsymbol{C} 2)$ then the functional $z \mapsto\|F(z)\|_{L^{2}}$ is coercive, i.e.

$$
\|F(z)\|_{L^{2}} \rightarrow \infty \text { whenever }\|z\|_{A C_{0}^{2}} \rightarrow \infty .
$$

Proof Let us take $m>8 B$ (cf. (C2)). By (19) and assumptions (C1)-(C2) we have

$$
\|F(z)\|_{L_{m}^{2}} \geq\left\|z_{x y}\right\|_{L_{m}^{2}}-\left(B\|z\|_{L_{m}^{2}}+B\left\|w_{0}\right\|_{L_{m}^{2}}+B\left\|w_{1}\right\|_{L_{m}^{2}}+B\left\|w_{2}\right\|_{L_{m}^{2}}\right)-D,
$$

where $D=2\|b\|_{L_{m}^{2}}$. By Lemma 1 and thanks to (12) it follows that

$$
\|F(z)\|_{L_{m}^{2}} \geq\|z\|_{A C_{0, m}^{2}}-\frac{8 B}{m}\|z\|_{A C_{0, m}^{2}}-D=\|z\|_{A C_{0, m}^{2}}\left(1-\frac{8 B}{m}\right)-D .
$$

Inequality $m>8 B$ implies that $\|F(z)\|_{L_{m}^{2}} \rightarrow \infty$ if $\|z\|_{A C_{0, m}^{2}} \rightarrow \infty$. Since the pairs of the norms $\|\cdot\|_{L^{2}},\|\cdot\|_{L_{m}^{2}}$ and $\|\cdot\|_{A C_{0}^{2}},\|\cdot\|_{A C_{0, m}^{2}}$ are equivalent, we conclude that (20) holds.

Let $\left\{z^{k}\right\}_{k=0}^{\infty} \subset A C_{0}^{2}$ be an arbitrary sequence. Denote by $\left\{g^{k}\right\} \subset L^{2}\left(Q, \mathbb{R}^{n}\right)$ a sequence of functions defined by

$$
\begin{aligned}
g^{k}(x, y)= & f^{1}\left(x, y, z^{k}(x, y)\right) \\
& +\int_{0}^{x} \int_{0}^{y}\left(f^{2}\left(s, t, z^{k}(s, t)\right)+A^{1}(s, t) z_{x}^{k}(s, t)+A^{2}(s, t) z_{y}^{k}(s, t)\right) d s d t \\
& -v(x, y),
\end{aligned}
$$

for $k=0,1,2, \ldots$.

\section{Lemma 3 If}

1. the functions $f^{1}, f^{2}, A^{1}, A^{2}$ satisfy assumptions (C1) and (C2);

2. the sequence $\left\{z^{k}\right\}_{k=0}^{\infty} \subset A C_{0}^{2}\left(Q, \mathbb{R}^{n}\right)$ tends to $z^{0} \in A C_{0}^{2}\left(Q, \mathbb{R}^{n}\right)$ weakly in $A C_{0}^{2}\left(Q, \mathbb{R}^{n}\right)$ then

(a) the sequence of functions $\left\{z^{k}\right\}$ tends uniformly to $z^{0}$ on the interval $Q$;

(b) the sequence $\left\{g^{k}\right\}$ tends to $g^{0}$ for $(x, y) \in Q$ a.e.

Moreover, there exists a function $b^{0} \in L^{2}\left(Q, \mathbb{R}^{+}\right)$such that

$$
\left|g^{k}(x, y)\right| \leq b^{0}(x, y)
$$

for a.e. $(x, y) \in Q$ and $k=1,2, \ldots$

Proof We first prove that the weak convergence of the sequence $\left\{z^{k}\right\}$ to $z^{0}$ in the space $A C_{0}^{2}\left(Q, \mathbb{R}^{n}\right)$ implies the uniform convergence of the sequence $\left\{z^{k}\right\}$ to $z^{0}$ on the interval $Q$. By the definition of the inner product [see (10)] the weak convergence of the sequence $\left\{z^{k}\right\}$ to $z^{0}$ in the space $A C_{0}^{2}\left(Q, \mathbb{R}^{n}\right)$ is equivalent to the weak convergence of mixed second order derivatives $\left\{z_{x y}^{k}\right\}$ to $z_{x y}^{0}$ in the space $L^{2}\left(Q, \mathbb{R}^{n}\right)$. Without loss of generality we can assume that $z^{0}=0$. Suppose that $z^{k}$ does not converge uniformly to $z^{0}=0$ while it converges to 0 weakly in $A C_{0}^{2}\left(Q, \mathbb{R}^{n}\right)$. Therefore, there exists $\varepsilon_{0}>0$ such that for any $n \in \mathbb{N}$ there is a point $\left(x^{n}, y^{n}\right) \in Q$ such that

$$
\left|z^{n}\left(x^{n}, y^{n}\right)\right|>\varepsilon_{0} .
$$


The sequence $\left\{\left(x^{n}, y^{n}\right)\right\} \subset Q$ is compact. Passing if necessary to a subsequence we can assume, that $\left(x^{n}, y^{n}\right)$ tends to some $(\tilde{x}, \tilde{y}) \in Q$. Denote by $\chi^{n}$ the characteristic function of the interval ${ }^{2}$

$$
\left\{(x, y) \in Q: 0 \leq x<x^{n}, 0 \leq y<y^{n}\right\}
$$

and by $\tilde{\chi}$ the characteristic function of the interval

$$
\{(x, y) \in Q: 0 \leq x<\tilde{x}, 0 \leq y<\tilde{y}\} .
$$

It is easy to notice that $\chi^{n}$ tends to $\tilde{\chi}$ on $Q$ a.e. This implies the following inequalities

$$
\begin{aligned}
\lim _{n \rightarrow \infty}\left|z^{n}\left(x^{n}, y^{n}\right)\right| \leq & \lim _{n \rightarrow \infty}\left|z^{n}\left(x^{n}, y^{n}\right)-z^{n}(\tilde{x}, \tilde{y})\right|+\lim _{n \rightarrow \infty}\left|z^{n}(\tilde{x}, \tilde{y})\right| \\
= & \lim _{n \rightarrow \infty}\left|\int_{0}^{1} \int_{0}^{1} \chi^{n}(s, t) z_{x y}^{n}(s, t) d s d t-\int_{0}^{1} \int_{0}^{1} \tilde{\chi}(s, t) z_{x y}^{n}(s, t) d s d t\right| \\
& +\lim _{n \rightarrow \infty}\left|\int_{0}^{1} \int_{0}^{1} \tilde{\chi}(s, t) z_{x y}^{n}(s, t) d s d t\right| .
\end{aligned}
$$

Since $z_{x y}^{n}$ tends to zero weakly in $L^{2}\left(Q, \mathbb{R}^{n}\right)$ the last limit is equal zero. Therefore

$$
\begin{aligned}
\lim _{n \rightarrow \infty}\left|z^{n}\left(x^{n}, y^{n}\right)\right| & \leq \lim _{n \rightarrow \infty} \int_{0}^{1} \int_{0}^{1}\left|\chi^{n}(s, t)-\tilde{\chi}(s, t)\right|\left|z_{x y}^{n}(s, t)\right| d s d t \\
& \leq \lim _{n \rightarrow \infty}\left(\int_{0}^{1} \int_{0}^{1}\left|\chi^{n}(s, t)-\tilde{\chi}(s, t)\right|^{2} d s d t\right)^{\frac{1}{2}} \cdot\left(\int_{0}^{1} \int_{0}^{1}\left|z_{x y}^{n}(s, t)\right|^{2} d s d t\right)^{\frac{1}{2}} \\
& \leq C \cdot \lim _{n \rightarrow \infty}\left(\int_{0}^{1} \int_{0}^{1}\left|\chi^{n}(s, t)-\tilde{\chi}(s, t)\right|^{2} d s d t\right)^{\frac{1}{2}}=0
\end{aligned}
$$

where $C>0$ is some constant such that $\left\|z_{x y}^{n}\right\| \leq C$. Consequently, $\lim _{n \rightarrow \infty}\left|z^{n}\left(x^{n}, y^{n}\right)\right|=0$. This contradicts our assumption (22). Thus $z^{k}$ tends to $z^{0}$ uniformly on $Q$.

Next we prove the assertion (b) of Lemma 3. By assumptions (C1) the functions $f^{1}$ and $f^{2}$ are continuous with respect to the third variable; assumption (C2) allows to find an appropriate dominating integrable function and to apply Lebesgue's dominated convergence theorem, consequently we get that

$$
\lim _{k \rightarrow \infty} f^{1}\left(x, y, z^{k}(x, y)\right)=f^{1}\left(x, y, z^{0}(x, y)\right)
$$

for a.e. $(x, y) \in Q$ and

$$
\lim _{k \rightarrow \infty} \int_{0}^{x} \int_{0}^{y} f^{2}\left(s, t, z^{k}(s, t)\right) d s d t=\int_{0}^{x} \int_{0}^{y} f^{2}\left(s, t, z^{0}(s, t)\right) d s d t
$$

for a.e. $(x, y) \in Q$. Thanks to the fact that $z^{k}$ tends uniformly to $z^{0}$ the sequence $\left\{z^{k}(x, y)\right\}$ is uniformly bounded, therefore there exists $\bar{C}>0$ such that

$$
\left|z^{k}(x, y)\right| \leq \bar{C}
$$

2 the characteristic function $\chi$ of a set $X \subset \mathbb{R}^{n}$ is the function such that $\chi(x)=1 \Leftrightarrow x \in X$ and $\chi(x)=0$ otherwise. 
for $(x, y) \in Q$ and $k=1,2, \ldots$ Taking into account the above and again (C2), we get that there exists a function $b^{1} \in L^{2}\left(Q, \mathbb{R}^{+}\right)$such that

$$
\left|f^{1}\left(x, y, z^{k}(x, y)\right)\right|,\left|\int_{0}^{x} \int_{0}^{y} f^{2}\left(s, t, z^{k}(s, t)\right) d s d t\right| \leq b^{1}(x, y)
$$

for a.e. $(x, y) \in Q$ and $k=1,2, \ldots$ Integrating by parts, thanks (C1) and Fubini's theorem, we obtain

$$
\begin{aligned}
\int_{0}^{x} \int_{0}^{y} A^{1}(s, t) z_{x}^{k}(s, t) d s d t & =\int_{0}^{y}\left(\int_{0}^{x} A^{1}(s, t) z_{x}^{k}(s, t) d s\right) d t \\
& =\int_{0}^{y} A^{1}(x, t) z^{k}(x, t) d t-\int_{0}^{x} \int_{0}^{y} A_{x}^{1}(s, t) z^{k}(s, t) d s d t
\end{aligned}
$$

for $k=0,1, \ldots$. Since $z^{k}$ tends to $z^{0}$ uniformly on $Q$ provided that $z^{k}$ converges to $z^{0}$ weakly in $A C_{0}^{2}\left(Q, \mathbb{R}^{n}\right)$, we get by (25) that

$$
\begin{aligned}
\lim _{k \rightarrow \infty} \int_{0}^{x} \int_{0}^{y} A^{1}(s, t) z_{x}^{k}(s, t) d s d t \\
=\int_{0}^{y} \lim _{k \rightarrow \infty} A^{1}(x, t) z^{k}(x, t) d t-\int_{0}^{x} \int_{0}^{y} \lim _{k \rightarrow \infty} A_{x}^{1}(s, t) z^{k}(s, t) d s d t \\
=\int_{0}^{y} A^{1}(x, t) z^{0}(x, t) d t-\int_{0}^{x} \int_{0}^{y} A_{x}^{1}(s, t) z^{0}(s, t) d s d t \\
=\int_{0}^{x} \int_{0}^{y} A^{1}(s, t) z_{x}^{0}(s, t) d s d t
\end{aligned}
$$

for $(x, y) \in Q$. Similarly, we can show that

$$
\lim _{k \rightarrow \infty} \int_{0}^{x} \int_{0}^{y} A^{2}(s, t) z_{y}^{k}(s, t) d s d t=\int_{0}^{x} \int_{0}^{y} A^{2}(s, t) z_{y}^{0}(s, t) d s d t
$$

for $(x, y) \in Q$. By (25), (24) and thanks to (C1) we have

$$
\begin{aligned}
& \left|\int_{0}^{x} \int_{0}^{y} A^{1}(s, t) z_{x}^{k}(s, t) d s d t\right| \\
& \quad \leq \int_{0}^{y}\left|A^{1}(x, t)\right| \cdot\left|z^{k}(x, t)\right| d t+\int_{0}^{x} \int_{0}^{y}\left|A_{x}^{1}(s, t)\right| \cdot\left|z^{k}(s, t)\right| d s d t \\
& \quad \leq \int_{0}^{y} B \bar{C} d t+\int_{0}^{x} \int_{0}^{y} B \bar{C} d s d t \leq B \bar{C} \int_{0}^{1} d t+B \bar{C} \int_{0}^{1} \int_{0}^{1} d s d t=C_{1}
\end{aligned}
$$

for some constant $C^{1}>0$, all $(x, y) \in Q$ and $k=1,2, \ldots$. Similar bounds can be found for the integral $\int_{0}^{x} \int_{0}^{y} A^{2}(s, t) z_{y}^{k}(s, t) d s d t$. From (23), (26), (27) and (28) it follows that

$$
\lim _{k \rightarrow \infty} g^{k}(x, y)=g^{0}(x, y)
$$

for a.e. $(x, y) \in Q$. Moreover, there exists a function $b^{0} \in L^{2}\left(Q, \mathbb{R}^{+}\right)$such that $\left|g^{k}(x, y)\right| \leq b^{0}(x, y)$ for $k=1,2, \ldots$ and a.e. $(x, y) \in Q$. This completes the proof. 


\section{Main result and example}

Let us consider a functional $\varphi: A C_{0}^{2} \rightarrow \mathbb{R}$ given by the formula

$$
\varphi(z)=\frac{1}{2}\|F(z)-v\|_{L^{2}}^{2},
$$

where $F$ is the operator defined by (19) and $v$ is a fixed function from the space $L^{2}\left(Q, \mathbb{R}^{n}\right)$. We begin by proving some lemmas.

Lemma 4 If the functions $f^{1}, f^{2}, A^{1}, A^{2}$ satisfy assumptions(C1)-(C2), then the functional $\varphi$ given by (29) satisfies (PS)_condition.

Proof Let $\left\{z^{k}\right\} \subset A C_{0}^{2}$ be an arbitrary (PS) - sequence for the functional $\varphi$. By Lemma $2 \varphi$ is coercive. It implies that the sequence $\left\{z^{k}\right\}$ is weakly compact in $A C_{0}^{2}$. Passing if necessary to a subsequence we can assume, that $z^{k}$ tends to some $z^{0}$ weakly in $A C_{0}^{2}$. We claim that $\left\{z^{k}\right\}$ is compact with respect to the norm topology of the space $A C_{0}^{2}$. Thanks to assumptions (C1)-(C2) it is easy to check that the functional $\varphi$ is Fréchet differentiable and

$$
\begin{aligned}
\left\langle\varphi^{\prime}\left(z^{k}\right), h\right\rangle= & \int_{0}^{1} \int_{0}^{1}\left\langle h_{x y}(x, y)+f_{z}^{1}\left(x, y, z^{k}(x, y)\right) h(x, y)\right. \\
& +\int_{0}^{x} \int_{0}^{y}\left(f_{z}^{2}\left(s, t, z^{k}(s, t)\right) h(s, t)+A^{1}(s, t) h_{x}(s, t)\right. \\
& \left.\left.+A^{2}(s, t) h_{y}(s, t)\right) d s d t, z_{x y}^{k}(x, y)+g^{k}(x, y)\right\rangle d x d y,
\end{aligned}
$$

where the sequence $\left\{g^{k}\right\} \subset L^{2}\left(Q, \mathbb{R}^{n}\right)$ is given by formula (21). Let us put $h^{k}-z^{k}-z^{0}$, $k=1,2, \ldots$. From (30) it follows that

$$
\begin{aligned}
\left\langle\varphi^{\prime}\left(z^{k}\right)-\varphi^{\prime}\left(z^{0}\right), z^{k}-z^{0}\right\rangle & =\left\langle z_{x y}^{k}-z_{x y}^{0}, h_{x y}^{k}\right\rangle+\sum_{i=1}^{5} V^{i}\left(z^{k}\right) \\
& =\left\|z^{k}-z^{0}\right\|_{A C_{0}^{2}}^{2}+\sum_{i=1}^{5} V^{i}\left(z^{k}\right),
\end{aligned}
$$

where

$$
\begin{aligned}
V^{1}\left(z^{k}\right)= & \left\langle z_{x y}^{k}-z_{x y}^{0}, g^{k}-g^{0}\right\rangle \\
= & \int_{0}^{1} \int_{0}^{1}\left\langle z_{x y}^{k}(x, y)-z_{x y}^{0}(x, y), g^{k}(x, y)-g^{0}(x, y)\right\rangle d x d y, \\
V^{2}\left(z^{k}\right)= & \int_{0}^{1} \int_{0}^{1}\left\langle f_{z}^{1}\left(x, y, z^{k}(x, y)\right)\left(z^{k}(x, y)-z^{0}(x, y)\right)\right. \\
& +\int_{0}^{x} \int_{0}^{y} f_{z}^{2}\left(s, t, z^{k}(s, t)\right)\left(z^{k}(s, t)-z^{0}(s, t)\right) d s d t, z_{x y}^{k}(x, y) \\
& \left.+g^{k}(x, y)\right\rangle d x d y, \\
V^{3}\left(z^{k}\right)= & -\int_{0}^{1} \int_{0}^{1}\left\langle f_{z}^{1}\left(x, y, z^{0}(x, y)\right)\left(z^{k}(x, y)-z^{0}(x, y)\right)\right. \\
& +\int_{0}^{x} \int_{0}^{y} f_{z}^{2}\left(s, t, z^{0}(s, t)\right)\left(z^{k}(s, t)-z^{0}(s, t)\right) d s d t, z_{x y}^{0}(x, y)
\end{aligned}
$$




$$
\begin{aligned}
& \left.-g^{0}(x, y)\right\rangle d x d y, \\
V^{4}\left(z^{k}\right)= & \int_{0}^{1} \int_{0}^{1}\left\langle\int_{0}^{x} \int_{0}^{y} A^{1}(s, t)\left(z_{x}^{k}(s, t)-z_{x}^{0}(s, t)\right) d s d t,\right. \\
& \left.z_{x y}^{k}(x, y)+g^{k}(x, y)\right\rangle d x d y \\
& +\int_{0}^{1} \int_{0}^{1}\left\langle\int_{0}^{x} \int_{0}^{y} A^{2}(s, t)\left(z_{y}^{k}(s, t)-z_{y}^{0}(s, t)\right) d s d t,\right. \\
V^{5}\left(z^{k}\right)= & \left.-\int_{0}^{k}(x, y)+g_{0}^{k}(x, y)\right\rangle d x d y, \\
& z_{x y}^{0}(x, y)+g_{0}^{x} \int_{0}^{y} A^{1}(s, t)\left(z_{x}^{k}(s, t)-z_{x}^{0}(s, t)\right) d s d t, \\
& -\int_{0}^{1} \int_{0}^{1}\left\langle\int_{0}^{x} \int_{0}^{y} A^{2}(s, t)\left(z_{y}^{k}(s, t)-z_{y}^{0}(s, t)\right) d s d t,\right. \\
& \left.z_{x y}^{0}(x, y)+g^{0}(x, y)\right\rangle d x d y .
\end{aligned}
$$

By the Cauchy-Schwarz inequality we have the following estimation

$$
\begin{aligned}
\left|V^{1}\left(z^{k}\right)\right|^{2} \leq & \int_{0}^{1} \int_{0}^{1}\left|z_{x y}^{k}(x, y)-z_{x y}^{0}(x, y)\right|^{2} d x d y . \\
& \int_{0}^{1} \int_{0}^{1}\left|g^{k}(x, y)-g^{0}(x, y)\right|^{2} d x d y .
\end{aligned}
$$

Since $z_{x y}^{k}-z_{x y}^{0}$ converges weakly to zero in $L^{2}\left(Q, \mathbb{R}^{n}\right)$, therefore there exists a constant $C>0$ such that

$$
\left|V^{1}\left(z^{k}\right)\right|^{2} \leq C \int_{0}^{1} \int_{0}^{1}\left|g^{k}(x, y)-g^{0}(x, y)\right|^{2} d x d y .
$$

By Lemma 2 and Lebesgue dominated convergence theorem it follows that $V^{1}\left(z^{k}\right) \rightarrow 0$ as $k \rightarrow \infty$. We have proved that $z^{k}(x, y)$ tends to $z^{0}(x, y)$ uniformly on $Q$ (see Lemma 2). Therefore, it is easy to notice that $V^{2}\left(z^{k}\right)$ and $V^{3}\left(z^{k}\right)$ converge to zero as $k \rightarrow \infty$.

Let us consider the functional $V^{4}$. By (25) we have

$$
\begin{aligned}
V^{4}\left(z^{k}\right)= & \int_{0}^{1} \int_{0}^{1}\left\langle\int_{0}^{y} A^{1}(x, t)\left(z^{k}(x, t)-z^{0}(x, t)\right) d t\right. \\
& -\int_{0}^{x} \int_{0}^{y} A_{x}^{1}(s, t)\left(z^{k}(s, t)-z^{0}(s, t)\right) d s d t, \\
& \left.z_{x y}^{k}(x, y)+g^{k}(x, y)\right\rangle d x d y \\
& +\int_{0}^{1} \int_{0}^{1}\left\langle\int_{0}^{x} A^{2}(s, y)\left(z^{k}(s, y)-z^{0}(s, y)\right) d s\right. \\
& -\int_{0}^{x} \int_{0}^{y} A_{y}^{2}(s, t)\left(z^{k}(s, t)-z^{0}(s, t)\right) d s d t,
\end{aligned}
$$




$$
\left.z_{x y}^{k}(x, y)+g^{k}(x, y)\right\rangle d x d y .
$$

Using the Cauchy-Schwarz inequality and Lemma 2 it is easy to show that $V^{4}\left(z^{k}\right) \rightarrow 0$ as $k \rightarrow \infty$.

Similar considerations can be applied to $V^{5}\left(z^{k}\right)$. Thus $\lim _{k \rightarrow \infty} \sum_{i=1}^{5} V^{i}\left(z^{k}\right)=0$.

Now, let us observe that

$$
\lim _{k \rightarrow \infty} \varphi^{\prime}\left(z^{k}\right)\left(z^{k}-z^{0}\right)=0
$$

because $\left\{z^{k}\right\}$ is the (PS)—sequence for the functional $\varphi$ and the sequence $\left\{z^{k}-z^{0}\right\}$ is bounded. Moreover,

$$
\lim _{k \rightarrow \infty} \varphi^{\prime}\left(z^{0}\right)\left(z^{k}-z^{0}\right)=0
$$

since $z^{k}$ tends weakly to $z^{0}$ in $A C_{0}^{2}$. Combining these equalities and (31) we conclude that

$$
\lim _{k \rightarrow \infty}\left\|z^{k}-z^{0}\right\|_{A C_{0}^{2}}^{2}=0 .
$$

This gives us the desired conclusion that the functional $\varphi$ given by (29) satisfies (PS) condition.

Next, we prove the following

Lemma 5 If the functions $f^{1}, f^{2}, A^{1}, A^{2}$ satisfy assumptions (C1)-(C3) then for any $v \in$ $L^{2}\left(Q, \mathbb{R}^{n}\right)$ there exists a unique solution $h_{v} \in A C_{0}^{2}$ to the system

$$
F^{\prime}\left(z^{0}\right) h=v,
$$

where the operator $F: A C_{0}^{2} \rightarrow L^{2}\left(Q, \mathbb{R}^{n}\right)$ is given by (19) and $z^{0} \in A C_{0}^{2}$ is an arbitrary function.

Proof Let us put

$$
h(x, y)=\int_{0}^{x} \int_{0}^{y} g(s, t) d s d t,
$$

where $g \in L^{2}\left(Q, \mathbb{R}^{n}\right)$. Substituting the above into (32) we obtain

$$
H g=v,
$$

where

$$
\begin{aligned}
H g(x, y)= & g(x, y)+f_{z}^{1}\left(x, y, z^{0}(x, y)\right) \cdot \int_{0}^{x} \int_{0}^{y} g(s, t) d s d t \\
& +\int_{0}^{x} \int_{0}^{y}\left(f_{z}^{2}\left(s, t, z^{0}(s, t)\right) \int_{0}^{s} \int_{0}^{t} g(\sigma, \tau) d \sigma d \tau\right. \\
& \left.+A^{1}(s, t) \int_{0}^{t} g(s, \tau) d \tau+A^{2}(s, t) \int_{0}^{s} g(\sigma, t) d \sigma\right) d s d t .
\end{aligned}
$$

Let us denote by $\tilde{H}$ the operator defined by

$$
\tilde{H} g=H g-g-v .
$$


We will restrict our investigation of the operator $\tilde{H}$ to the space $L_{m}^{2}\left(Q, \mathbb{R}^{n}\right)$. We prove that for sufficiently large $m>0$ the mapping $\tilde{H}$ is contracting with respect to the norm $\|\cdot\|_{L_{m}^{2}}$ defined by (13). Under assumptions (C2) and (C3), there exists a constant $d>0$ such that

$$
\begin{aligned}
& \left\|\tilde{H}\left(g^{1}-g^{2}\right)\right\|_{L_{m}^{2}} \\
& \leq d\left(\int_{0}^{1} \int_{0}^{1}\left(e^{-m(x+y)} \int_{0}^{x} \int_{0}^{y}\left|g^{1}(s, t)-g^{2}(s, t)\right|^{2} d s d t\right) d x d y\right)^{\frac{1}{2}} \\
& +d\left(\int_{0}^{1} \int_{0}^{1}\left(e^{-m(x+y)} \int_{0}^{x} \int_{0}^{y}\left(\int_{0}^{s} \int_{0}^{t}\left|\left(g^{1}(\sigma, \tau)-g^{2}(\sigma, \tau)\right)\right|^{2} d \sigma d \tau\right) d s d t\right) d x d y\right)^{\frac{1}{2}} \\
& +d\left(\int_{0}^{1} \int_{0}^{1}\left(e^{-m(x+y)} \int_{0}^{x} \int_{0}^{y}\left(\int_{0}^{t}\left|\left(g^{1}(s, \tau)-g^{2}(s, \tau)\right)\right|^{2} d \tau\right) d s d t\right) d x d y\right)^{\frac{1}{2}} \\
& +d\left(\int_{0}^{1} \int_{0}^{1}\left(e^{-m(x+y)} \int_{0}^{x} \int_{0}^{y}\left(\int_{0}^{s}\left|\left(g^{1}(\sigma, t)-g^{2}(\sigma, t)\right)\right|^{2} d \sigma\right) d s d t\right) d x d y\right)^{\frac{1}{2}} \\
& \leq 4 d\left(\int_{0}^{1} \int_{0}^{1}\left(e^{-m(x+y)} \int_{0}^{x} \int_{0}^{y}\left|g^{1}(s, t)-g^{2}(s, t)\right|^{2} d s d t\right) d x d y\right)^{\frac{1}{2}} .
\end{aligned}
$$

Integrating by parts twice, in much the same way as in the proof of inequality (Kaczorek 2001), we obtain

$$
\left\|\tilde{H}\left(g^{1}-g^{2}\right)\right\|_{L_{m}^{2}} \leq \frac{4 d}{m^{2}}\left\|g^{1}-g^{2}\right\|_{L_{m}^{2}} .
$$

Hence for sufficiently large $m$, i.e. $m>2 \sqrt{d}$, the operator $\tilde{H}$ is contracting and, consequently, has a unique fixed point. It means that, there exists exactly one point $g^{0} \in L^{2}\left(Q, \mathbb{R}^{n}\right)$ such that $g^{0}=\tilde{H} g^{0}$. By (33) we get $H g^{0}=v$ and it follows easily that a function $h_{v}$ given by

$$
h_{v}(x, y)=\int_{0}^{x} \int_{0}^{y} g^{0}(s, t) d s d t
$$

is a solution of (32) for fixed $v \in L^{2}\left(Q, \mathbb{R}^{n}\right)$.

We are now in a position to show the main result of the work.

Theorem 3 If the functions $f^{1}, f^{2}, A^{1}, A^{2}$ satisfy assumptions (C1)-(C3) then for any $v \in$ $L^{2}\left(Q, \mathbb{R}^{n}\right)$ the integro-differential system (7)-(8) has a unique solution $z_{v} \in A C_{0}^{2}$. The solution $z_{v}$ continuously depends on $v$ with respect to the norm topology in the spaces $L^{2}\left(Q, \mathbb{R}^{n}\right)$ and $A C_{0}^{2}$. Moreover, the operator

$$
L^{2}\left(Q, \mathbb{R}^{n}\right) \ni v \mapsto z_{v} \in A C_{0}^{2}
$$

is differentiable (in Fréchet sense).

Proof If follows from Lemmas 4 and 5 that the operator $F$ given by (19) meets assumptions of Theorem 1. Thus system (7)-(8) has a solution $z_{v}$ which satisfies the requirements of our theorem.

We now give an example of integro-differential system of the form (7)-(8) which satisfies assumptions of Theorem 3. For simplicity we put $n=1$. 
Example 1 Consider 2D integro-differential system

$$
\begin{aligned}
& z_{x y}(x, y)+w^{1}(x, y)\left(\frac{z^{3}(x, y)}{1+z^{2}(x, y)}+\psi^{1}(z(x, y))\right. \\
& +\int_{0}^{x} \int_{0}^{y}\left(w^{2}(s, t) \frac{z(s, t)-1}{1+z^{2}(x, y)}+\psi^{2}(z(x, y))\right. \\
& \left.+A^{1}(s, t) z_{x}(s, t)+A^{2}(s, t) z_{y}(s, t) d s d t\right)=v(x, y),
\end{aligned}
$$

where $w^{1}, w^{2}, A^{1}, A^{2}$ are some polynomials, $v \in L^{2}(Q, \mathbb{R})$ and $\psi^{1}, \psi^{2}$ are some $C^{1}$-class functions with unbounded derivatives. For example one can take $\psi^{1}(z)=\cos z^{k}$ and $\psi^{2}(z)=$ $\sin z^{l}$, where $k, l>1$. This simple and theoretical example allows us to emphasize the difference between our work and some other methods of nonlinear analysis.

It is easy to see that system (34) satisfies assumptions (C1)-(C3). Hence by Theorem 3 for any $v \in L^{2}(Q, \mathbb{R})$ there exists a solution $z_{v} \in A C_{0}^{2}$ to the system (34) with the following properties:

1. the solution $z_{v}$ is unique,

2. $z_{v}$ continuously depends on $v$ with respect to the norm topology of the spaces $L^{2}\left(Q, \mathbb{R}^{n}\right)$ and $A C_{0}^{2}$, i.e. system (34) is stable,

3. the operator $L^{2}(Q, \mathbb{R}) \ni v \mapsto z_{v} \in A C_{0}^{2}$ is differentiable in Fréchet sense, i.e. system (34) is robust.

Let us notice that the functions $f^{1}(x, y, z)=w^{1}(x, y)\left(\frac{z^{3}}{1+z^{2}}+\psi^{1}(z)\right)$ and $f^{2}(x, y, z)=w^{2}(x, y) \frac{z-1}{1+z^{2}}+\psi^{2}(z)$ are not Lipschitz functions $\left(\sin z^{l}\right.$ and $\cos z^{l}$ with $k, l \geq 1$ have "fast variation" when $|z| \rightarrow \infty)$ and consequently we cannot apply the Banach contraction principle. In this case the Schauder fixed point theory may be applicable. But even using sophisticated fixed point theorems we get only the existence of a solution to system (34) and can hardly say anything related to properties (1)-(3).

\section{Concluding remarks}

In the paper two-dimensional integro-differential system was investigated. The main result of this work is theorem 3 on the stability and robustness of a solution to considered system (7)-(8). As far as we know 2D integro-differential systems have not been studied before. Onedimensional integro-differential systems described by ordinary differential operators were examined in many works (see monogrph Lakshmikantham 1995 and references therein). It is important to notice that integro-differential operators can be used in mathematical modeling of systems with "memory", i.e. systems where the state at each moment $t$ depends on its behavior on some interval $\left[t_{0}, t\right)$. In our opinion $2 \mathrm{D}$ integro-differential systems have the potential to play a similar role.

Open Access This article is distributed under the terms of the Creative Commons Attribution 4.0 International License (http://creativecommons.org/licenses/by/4.0/), which permits unrestricted use, distribution, and reproduction in any medium, provided you give appropriate credit to the original author(s) and the source, provide a link to the Creative Commons license, and indicate if changes were made. 


\section{References}

Aubin, J.-P., \& Ekeland, I. (2006). Applied Nonlinear Analysis. New York: Dover Publications.

Berkson, E., \& Gillespie, T. A. (1984). Absolutely continuous functions of two variables and well-bounded operators. Journal of the London Mathematical Society (2), 30(2), 305-321.

Bielecki, A. (1956). Une remarque sur l'application de la méthode de Banach-Cacciopoli-Tikhonov dans la théorie de l'équation $s=f(x, y, z, p, q)$. Bull. Acad. Polon. Sci. Cl. III., 4, 265-268.

Bors, D., \& Walczak, S. (2012). Application of 2D systems to investigation of a process of gas filtration. Multidimensional Systems and Signal Processing, 23(1-2), 119-130.

Bracewell, R. N. (1995). Two dimensional imaging (1st ed.). Upper Saddle River, NJ: Prentice Hall.

Dey, A., \& Kar, H. (2011). LMI-based criterion for the robust stability of 2D discrete state-delayed systems using generalized overflow nonlinearities. Journal of Control Science and Engineering. doi:10.1155/ $2011 / 271515$.

Fornasini, E. (1991). A 2-D systems approach to river pollution modelling. Multidimensional Systems and Signal Processing, 2(3), 233-265.

Fornasini, E., \& Marchesini, G. (1976). State-space realization theory of two-dimensional filters. IEEE Transactions on Automatic Control, 21(4), 484-492.

Fukuda, S. (ed.) (2015). Emotional Engineering (Vol. 3). Springer International Publishing.

Galkowski, K., Lam, J., Xu, S., \& Lin, Z. (2003). LMI approach to state-feedback stabilization of multidimensional systems. International Journal of Control, 76(14), 1428-1436.

Idczak, D., \& Skowron, A. (2012). On the diffeomorphisms between Banach and Hilbert spaces. Advanced Nonlinear Studies, 12(1), 89-100.

Idczak, D., \& Walczak, S. (2000). On the existence of a solution for some distributed optimal control hyperbolic system. International Journal of Mathematics and Mathematical Sciences, 23(5), 297-311.

Kaczorek, T. (1985). Two-dimensional linear systems, volume 68 of Lecture Notes in Control and Information Sciences. Berlin: Springer.

Kaczorek, T. (2001). Perfect observers for singular 2-D Fornasini-Marchesini models. IEEE Transactions on Automatic Control, 46(10), 1671-1675.

Lakshmikantham, V. (1995). Theory of integro-differential equations (1st ed.). Lausanne: Gordon and Breach Science Publishers.

Limaye, B. V. (1996). Functional analysis. New Delhi: New Age International Publishers Limited.

Lomadze, V., Rogers, E., \& Wood, J. (2008). Singular 2D behaviors: homologies. Georgian Mathematical Journal, 15(1), 139-157.

Paszke, W., Lam, J., Gakowski, K., Xu, S., \& Lin, Z. (2004). Robust stability and stabilisation of 2d discrete state-delayed systems. Systems \& Control Letters, 51(34), 277-291.

Singh, V. (2008). Stability analysis of 2-D discrete systems described by the Fornasini-Marchesini Second model with state saturation. IEEE Transactions on Circuits and Systems II: Express Briefs, 55(8), 793796.

Šremr, J. (2010). Absolutely continuous functions of two variables in the sense of Carathodory. Electronic Journal of Differential Equations, 2010(154), 1-11.

Walczak, S. (1987). Absolutely continuous functions of several variables and their application to differential equations. Bulletin of the Polish Academy of Sciences Mathematics, 35(11-12), 733-744.

Walczak, S. (1998). On the differentiability of absolutely continuous functions of several variables, remarks on the Rademacher theorem. Bulletin of the Polish Academy of Sciences Mathematics, 36(9-10), 513-520.

Monika Bartkiewicz is a senior lecturer at the Faculty of Mathematics and Computer Science, University of Lodz, Poland. She received her Ph.D. degree in 2001 from the University of Lodz. Her research interests are optimal control problems described by ordinary and partial differential equations, stability and sensitivity of solutions and variational methods. 


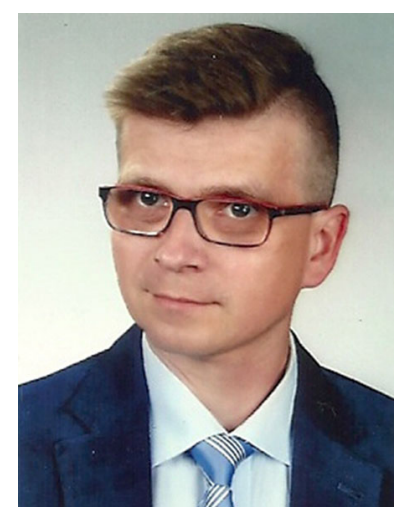

Marek Majewski is an assistant professor at the Faculty of Mathematics and Computer Science, University of Lodz, Poland. He received his Ph.D. degree in 2003 from the University of Lodz. His research interests are optimal control problems described by ordinary and partial differential equations, stability and sensitivity of solutions, continuous 2D control systems and fractional calculus.

Stanisław Walczak is a Full Professor in the Department of Mathematics and Computer Science at the University of Lodz (Poland). He received his PhD degree in 1972 from the University of Lodz and his Professor degree in 1986. His research interests include optimization theory and differential equations. 